\title{
Role of the cardiac nerve in the effect of a novel innocuous stimulus on the heart rate of Megalobulimus mogianensis
}

S.M.B. Romero ${ }^{1}$ and A. Hoffmann²
${ }^{1}$ Departamento de Biologia, Faculdade de Filosofia, Ciências e Letras de Ribeirão Preto, ${ }^{2}$ Departamento de Fisiologia, Faculdade de Medicina de Ribeirão Preto, Universidade de São Paulo, Ribeirão Preto, SP, Brasil
Correspondence

S.M.B. Romero

Departamento de Biologia

FFCLRP, USP

Av. Bandeirantes, 3900

14040-901 Ribeirão Preto, SP

Brasil

Fax: +55-16-3602-3666

E-mail: sbromero@ffclrp.usp.br

Publication supported by FAPESP.

Received June 17, 2005

Accepted March 24, 2006

\begin{abstract}
The effects of a brief jet of water delivered to the anterior portion of body-head on the heart rate of Megalobulimus mogianensis were determined in a group of intact snails $(\mathrm{N}=8)$, previously prepared for electrocardiogram recording. The heart rate was significantly increased following stimulation. Nevertheless, with repetition of the stimulus there was a significant decrease in the magnitude of the heart rate variation and in the time for the basal heart rate to recover (first stimulus, $7.4 \pm 1.2 \mathrm{bpm}$ and $15.5 \pm 1.8 \mathrm{~min}$; second stimulus, $4.8 \pm 1.0$ bpm and $10.6 \pm 1.5 \mathrm{~min}$; third stimulus, $5.0 \pm 0.3 \mathrm{bpm}$ and $11.1 \pm 1.8$ $\mathrm{min}$ ), indicating that this behavioral response undergoes early habituation. To determine the role of the cardiac nerve in mediating the heart rate alterations induced by the jet of water two other groups were tested: denervated animals $(\mathrm{N}=8)$ and sham-operated control animals $(\mathrm{N}=8)$. Although the innocuous stimulus caused the heart rate to increase significantly in both experimental groups, the mean increase in heart rate in denervated animals $(3.2 \pm 0.4 \mathrm{bpm})$ was $41 \%$ of the value obtained in sham-operated animals $(7.8 \pm 1.5 \mathrm{bpm})$, indicating that the cardiac nerve is responsible for $59 \%$ of the cardioacceleration induced by the innocuous stimulus. The increase in heart rate observed in denervated animals may be due to an increase in venous return promoted by the intense muscular activity associated with the retraction-protraction of the anterior part of the body induced by the jet of water.
\end{abstract}

It is known that rain, after a drought, stimulates the aestivating snail to emerge from its shell and that non-aestivating pulmonates tend to increase their behavioral activity during or following daytime rain (1). In addition, we observed that the stimulation by a brief directed jet of water causes the retracted snail to emerge from its shell,
Key words

- Cardiac nerve

- Heart rate

- Snail

- Megalobulimus mogianensis
- Innocuous stimulus whereas completely extended animals (still or in locomotion) exhibit a withdrawal reaction followed by an acceleration of the movements (Romero SMB and Hoffmann A, unpublished data). These behavioral alterations reflect changes in the level of arousal, implying an increase in the general activity level and responsiveness of the animal (2). Since 
molluscs have an open-circulatory system and therefore large hemocoelic sinuses, the contraction of body wall musculature changes the distribution of hemolymph within the cephalopedal hemocoel and indirectly affects cardiac activity (3). Thus, behaviors associated with intense muscular activity such as withdrawal reflexes and locomotion are in general associated with concomitant changes in heart rate (4-6).

Several aspects of the defense reaction evoked by a novel (innocuous or noxious) stimulus have been studied in various gastropod species by a number of investigators (5, 7-10). Although the pathways which control defensive behaviors often share circuitry with cardiovascular function $(11,12)$, the literature concerning the heart rate alterations associated with these behaviors is limited. The effects of the mechanical stimulation of the tail and head on the heart rate of the slug Limax maximus were studied by MacKay and Gelperin (13) and Krontiris-Litowitz (5) reported the effects of cutaneous stimulation on the cardiovascular function of Aplysia californica.

Studies have been conducted in our laboratory to determine the effects of different external and/or internal factors and behavior on the heart rate of the terrestrial snail Megalobulimus mogianensis as well as to establish the relative contribution of intrinsic and neural control $(3,4,14,15)$. Thus, the objective of the present investigation was to extend previous studies by investigating the effects of an innocuous stimulus represented by a jet of water on the heart rate of these snails and determining the role of the heart innervation in mediating the alterations.

Adult specimens (103-153 g) of $M$. mogianensis Simone and Leme (=M. sanctipauli sensu Romero and Hoffmann nonPilsbry and Ihering 1900) collected at Santa Rita do Passa Quatro (21 ${ }^{\circ} 40^{\prime} \mathrm{S}, 47^{\circ} 30^{\prime} \mathrm{W}$ ), State of São Paulo, Brazil, were used. The animals were kept in a terrarium and fed green leaves ad libitum once a day. All animals were deprived of food for 4-6 days before the experiments.

To record heart rate, three silver chloride electrodes were chronically implanted into the visceral mass of the animals according to the technique described by Romero and Hoffmann (16). Polygraphic recordings were obtained at a room temperature of $25^{\circ} \mathrm{C}$, with a Nihon Kohden polygraph model RM6100 (Nihon Kohden Corporation, Tokyo, Japan). The animals were individually placed in plastic containers $(29 \times 29 \times 14 \mathrm{~cm})$ and transferred to the special room about $3 \mathrm{~h}$ before the beginning of the experiments in order to obtain a regular heart rate. All animals were prepared for electrocardiogram recording $48 \mathrm{~h}$ before the experiments.

The heart rate of completely extended (still or in locomotion) snails was recorded for about $3 \mathrm{~min}$ to evaluate the effects of the innocuous stimulus on cardiac function. Then, a brief jet of water was applied to the anterior portion of the body-head. Reproducible stimuli were delivered by pressing totally and in a single movement a wash bottle of $500 \mathrm{~mL}$ placed at a distance of 20 $\mathrm{cm}$ from the animal, with a U-shaped 13-mm wide aluminum device applied to its central part. Heart rate was continuously recorded before, during and after stimulation until recovery of basal heart rate. We considered the heart rate obtained post-stimulation to be the highest heart rate recorded after application of the jet of water (1-3 min, in 22 animals). Heart rate data are reported as beats per min (bpm).

We first determined how the innocuous stimulus caused the heart rate to vary in a group of intact animals $(\mathrm{N}=8)$. Each animal was submitted to three stimulations. To prevent probable anticipatory cardiac responses (see Gianaros and Quigley, 17), a variable interval between stimulations was employed corresponding to the minimal time necessary for basal heart rate to recover (8 to 25 $\min )$. In order to investigate the role of the nervous system in mediating these heart rate 
alterations, two other groups were tested: denervated animals $(\mathrm{N}=8)$ submitted to section of the cardiac nerve (see Romero and Hoffmann, 18) and sham-operated control animals $(\mathrm{N}=8)$ submitted to the same surgical procedure except that the nerve was only localized but left intact. Operated animals were submitted to experimental protocols 8 to 14 days after surgery. Each animal was submitted to only one stimulation. At the end of the experiments each animal was dissected to visually confirm if the nerve had really been severed.

Data from repeated measures made on the same group of animals under different conditions were analyzed statistically by oneway analysis of variance for repeated measures followed by the Newman-Keuls test. Data from two measurements made on the same group of animals were analyzed statistically by the two-tailed paired Student $t$ test, whereas comparisons between two different groups of animals were made by the two-tailed Student $t$-test. Associations between two quantitative variables were explored by Pearson coefficient correlation. All comparisons having a probability $<0.05$ were considered to be significant.

In intact snails the application of a brief jet of water on the anterior portion of the body induces a withdrawal reflex causing the head, the buccal fringes and the tentacles to retract into the mass of the body, a process that is followed by the liberation of the retracted parts. The entire sequence took 23 to $70 \mathrm{~s}$. All the stimulated animals showed an increase in heart rate $(\mathrm{P}=0.00039)$ with mean values ranging from $32.7 \pm 1.1$ (basal heart rate) to $40.1 \pm 0.9 \mathrm{bpm}$ (heart rate poststimulation). Nevertheless, when the stimulus was repeatedly applied there was a decrease in the magnitude of the increase in heart rate (Figure 1). The increase in heart rate induced by the first stimulus $(7.4 \pm 1.2$ $\mathrm{bpm}$ ) differed significantly from the increases induced by the second and third ones ( $4.8 \pm$ 1.0 and $5.0 \pm 0.3 \mathrm{bpm}$, respectively), whose values did not differ significantly from one to another. Similarly, the time for the basal heart rate to recover associated with the first stimulus $(15.5 \pm 1.8 \mathrm{~min})$ differed significantly from the time associated with the subsequent two stimuli (10.6 \pm 1.5 and 11.1 $\pm 1.8 \mathrm{~min}$ ) which did not differ significantly from one to another. Thus, we used only the heart rate associated with the first stimulation, which was the highest, to evaluate the role of the cardiac nerve in mediating the heart rate increases.

Although the mean time for head retraction-protraction decreased during three stimulations $(38 \pm 4,37 \pm 6$, and $35 \pm 4$ s) the differences were not statistically significant (Figure 1, inset). This was probably due to the high values of the standard error of the mean.

Figure 2 shows the effect of the innocuous stimulus on the mean heart rate of denervated $(\mathrm{N}=8)$ and sham-operated control animals $(\mathrm{N}=8)$. Although the innocuous stimulus caused the heart rate to increase significantly in both experimental groups

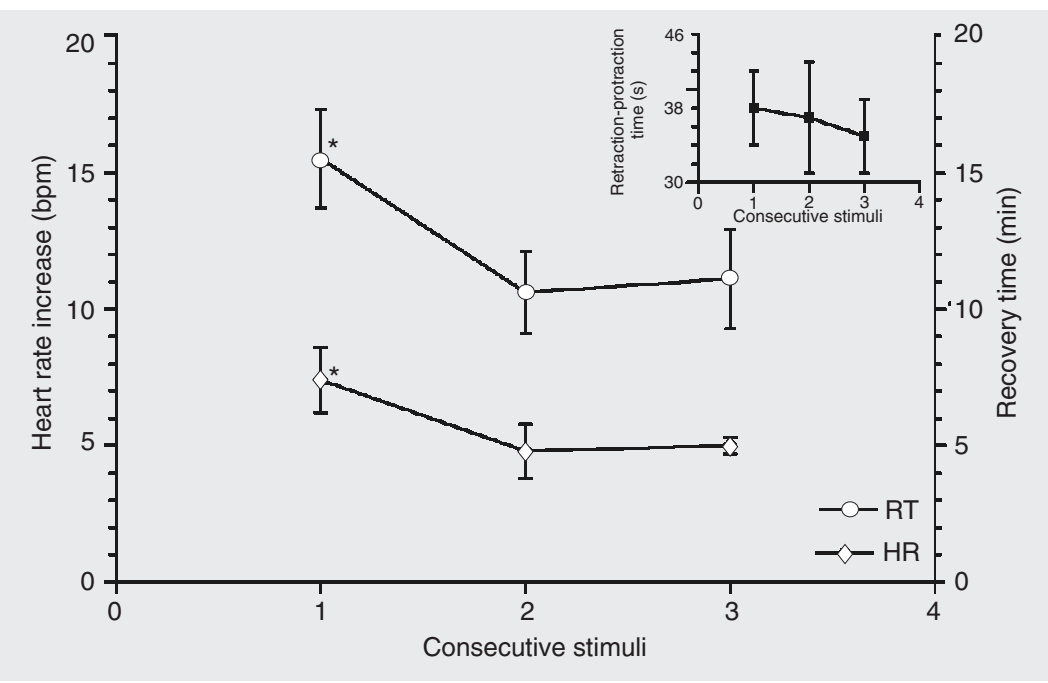

Figure 1. Effect of the repetition of stimuli on mean heart rate increase (lozenges) and mean time for recovery of basal heart rate (circles) in intact specimens of Megalobulimus mogianensis. Heart rate data are reported as bpm and recovery time is reported as min at $25^{\circ} \mathrm{C}$. Data are reported as mean \pm SEM for 8 specimens. The inset graph indicates the mean time in seconds of retraction-protraction of the body-head as a function of consecutive stimuli. $\mathrm{RT}=$ recovery time; $\mathrm{HR}=$ heart rate. ${ }^{*} \mathrm{P}<0.05$ compared to stimuli 2 and 3 (ANOVA for repeated measures followed by the Newman-Keuls test). 
(from $33.4 \pm 1.4$ to $36.6 \pm 1.7 \mathrm{bpm}$ in the denervated group, $\mathrm{P}=0.00016$; from $32.2 \pm$ 1.1 to $40.0 \pm 1.0 \mathrm{bpm}$ in the sham-operated group, $\mathrm{P}=0.00105$ ) the increase in heart rate obtained in denervated animals differed significantly from that obtained in sham-operated animals $(\mathrm{P}=0.0098)$. The mean increase in heart rate in denervated animals $(3.2 \pm 0.4 \mathrm{bpm})$ was $41 \%$ of the value obtained in sham-operated animals $(7.8 \pm 1.5$ bpm), indicating that the cardiac nerve is responsible for $59 \%$ of the increase in heart rate induced by the innocuous stimulus. A significant positive correlation $(\mathrm{P}=0.0466)$ between the time for head retraction-protraction and the increase in heart rate was detected in denervated snails.

Our results indicate that stimulating the head of M. mogianensis with a jet of water induces a withdrawal reaction consisting of retraction-protraction of the anterior part of the head-foot, which is always associated with an increase in heart rate. MacKay and Gelperin (13) observed that mechanical caudal stimulation of the slug L. maximus elicited cardioacceleration accompanied by locomotion. Similar stimulation of the head produced cardioinhibition which outlasted the stimulation and was sometimes accompanied by a transient rebound excitation. The increase in heart rate associated with the increase in activity confers an adaptive advantage to the animal since during exercise the blood must deliver more oxygen to the tissues. This can be achieved through an increase of the oxygen extraction from the blood per circuit or by an increase of the

Figure 2. Mean heart rate increase associated with the delivery of a jet of water to the headbody of sham-operated and denervated specimens of Megalobulimus mogianensis. Heart rate data are reported as bpm at $25^{\circ} \mathrm{C}$. Data are reported as mean \pm SEM for 8 specimens in each group. ${ }^{*} P=0.001$ compared to sham-operated animals (Student t-test).

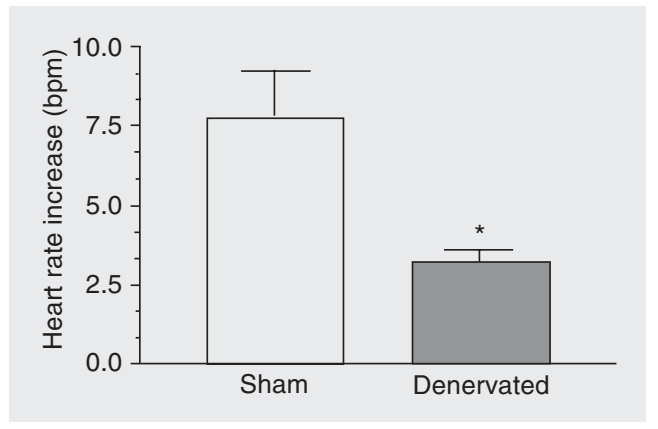

cardiac output resulting from an increase of the heart rate and/or of the stroke volume (19).

Nevertheless, repetition of the stimulus induced a gradual decrease in the magnitude of the heart rate increase, indicating that this behavioral response undergoes early habituation. MacKay and Gelperin (13) also observed in $L$. maximus that the stimuli lose efficacy with repetition. In A. californica, when the gill-withdrawal reflex was repeatedly evoked by an innocuous stimulus applied to the siphon or mantle shelf, the amplitude of the response showed a marked decrease (7-9). According to Kandel (9), when an animal is exposed to a novel stimulus it first responds with a combination of orienting and defensive reflexes, but if the stimulus proves to be unrewarding or innocuous, the animal will reduce and ultimately suppress its responses to it. Thus, habituation serves to decrease the response to a stimulus whose informational value has decreased as a result of its inconsequential repetition (20). As stated earlier, the first jet of water probably increased the level of arousal of Megalobulimus, making it unnecessary to respond with the same strength to the other two stimuli. A decrease in the strength of the response when the initially novel stimulus is presented repeatedly confers an adaptive advantage since it reduces energy expenditure and frees the animals to attend to stimuli that are more significant for survival.

Our investigations of the role of the nervous system in mediating the heart rate alterations revealed that the stimulation by a jet of water induces significant increases in heart rate in both sham-operated and denervated animals although the mean increase in the denervated animals was significantly lower than that observed in the snails with an intact cardiac nerve. Our results indicate that the cardiac nerve is responsible for $59 \%$ of the increase in heart rate induced by the innocuous stimulus. In agreement with these re- 
sults, Krontiris-Litowitz (5) showed that sensitizing stimulation of the body wall of $A$. californica causes a long-term increase in heart rate which is prevented by chemical blockade of the connectives that provide communication between the sensory neurons in the pleural ganglion and the cardiovascular command neurons in the abdominal ganglion. As is known, the nervous system simultaneously activates the motor behavioral patterns and the correlated vegetative adjustments. Thus, a neurally induced increase in heart rate represents the contribution of the nervous system to the increase in the level of activity induced by the innocuous stimulus. On the other hand, the increase in heart rate observed in denervated animals is expected since the jet of water induces a vigorous activity consisting of retraction-protraction of the anterior part of the body. The retraction of the head may generate an increase in the cephalopedal hemocoel pressure and consequently an increase in venous return which is responsible for the increase in heart rate (3). The longer the time in which the head remains retracted pressing the cephalopedal sinus, the higher the venous return. We detected a significant positive correlation between the time for head retraction-protraction and the increase in heart rate in denervated snails which suports this view.

\section{References}

1. Prior DJ. Water-regulatory behaviour in terrestrial gastropods. Biol Res 1985; 60: 403-424.

2. Dieringer N, Koester J, Weiss KR. Adaptive changes in heart rate of Aplysia californica. J Comp Physiol 1978; 123: 11-21.

3. Romero SMB, Hoffmann A. Role of the visceral nerve in heart rate variations during different behavioral patterns in Megalobulimus sanctipauli (Mollusca, Gastropoda, Pulmonata). Comp Biochem Physiol 1992; 103A: 93-98.

4. Romero SMB, Hoffmann A. Heart rate and behavioral patterns of Megalobulimus sanctipauli (Mollusca, Gastropoda, Pulmonata). Braz J Med Biol Res 1991; 24: 223-227.

5. Krontiris-Litowitz J. Sensitizing stimulation causes a long-term increase in heart rate in Aplysia californica. J Comp Physiol [A] 1999; 185: 181-186.

6. Marshall DJ, Peter R, Chown SL. Regulated bradycardia in the pulmonate limpet Siphonaria (Gastropoda: Mollusca) during pollutant exposure: implication for biomarker studies. Comp Biochem Physiol A Mol Integr Physiol 2004; 139: 309-316.

7. Pinsker H, Kupfermann I, Castellucci V, Kandel E. Habituation and dishabituation of the gill-withdrawal reflex in Aplysia. Science 1970; 167: $1740-1742$.

8. Peretz B, Jacklet JW, Lukowi K. Habituation of reflexes in Aplysia: contribution of the peripheral and central nervous systems. Science 1976; 191: 396-399.

9. Kandel E. Small systems of neurons. Sci Am 1979; 241: 66-76.

10. Romero SMB, Hoffmann A, Menescal-de-Oliveira L. Is there an opiate receptor in the snail Megalobulimus sanctipauli? Action of morphine and naloxone. Comp Biochem Physiol 1994; 107: 37-40.

11. Alevizos A, Bailey $\mathrm{CH}$, Chen M, Koester J. Innervation of vascular and cardiac muscle of Aplysia by multimodal motoneuron L7. $J$ Neurophysiol 1989; 61: 1053-1063.

12. Cleary LJ, Byrne JH. Identification and characterization of a multifunction neuron contributing to defensive arousal in Aplysia. $J$ Neurophysiol 1993; 70: 1767-1776.

13. Mackay AR, Gelperin A. Pharmacology and reflex responsiveness of the heart in the giant garden slug, Limax maximus. Comp Biochem Physiol 1972; 43A: 877-896.

14. Romero SMB, Hoffmann A. Heart rate and temperature in the snail Megalobulimus sanctipauli: role of the cardiac nerve. Can J Physiol Pharmacol 1996; 74: 1362-1365.

15. Rizzatti ACS, Romero SMB. Heart rate and body weight alterations in juvenile specimens of the tropical land snail Megalobulimus sanctipauli during dormancy. Braz J Med Biol Res 2001; 34: 959-967.

16. Romero SB, Hoffmann A. A technique for recording the electrocardiogram of Megalobulimus sp (Mollusca: Gastropoda: Pulmonata) during activity. Comp Biochem Physiol 1988; 90A: 115-116.

17. Gianaros PJ, Quigley KS. Autonomic origins of a nonsignal stimulus-elicited bradycardia and its habituation in humans. Psychophysiology 2001; 38: 540-547.

18. Romero SMB, Hoffmann A. A technique for cardiac denervation in Megalobulimus sanctipauli (Mollusca, Gastropoda, Pulmonata). Braz J Med Biol Res 1991; 24: 1185-1188.

19. Smith PJS. Cardiac output in the Mollusca: Scope and regulation. Experientia 1987; 43: 956-965.

20. Prescott SA. Interactions between depression and facilitation within neural networks: updating the dual-process theory of plasticity. Learn Mem 1998; 5: 446-466. 